\title{
Low birth weight and fetal anaemia as risk factors for infant morbidity in rural Malawi Boniface Kalanda $^{1,2}$, Francine Verhoeff ${ }^{1}$, Saskia le Cessie ${ }^{3}$, John Brabin ${ }^{1,4}$
}

1. Child and Reproductive Health Group, Liverpool School of Tropical Medicine, Liverpool, United Kingdom.

2. College of Medicine, University of Malawi, Blantyre, Malawi.

3. Department of Medical Statistics, Leiden University Medical Centre, The Netherlands.

4. Department of Paediatrics, Academic Medical Centre, University of Amsterdam, The Netherlands.

Correspondence: Professor B.J Brabin, Child and Reproductive Health Group, Liverpool School of Tropical Medicine, Pembroke Place, Liverpool L3 5QA, England, United Kingdom Email: b.j.brabin@kiv. ac.uk

\section{Abstract}

Low birth weight (LBW) and fetal anaemia (FA) are common in malaria endemic areas. To investigate the incidence of infectious morbidity in infants in rural Malawi in relation to birth weight and fetal anaemia, a cohort of babies was followed for a year on the basis of LBW $(<2500)$ and FA (cord haemoglobin $<12.5 \mathrm{~g} / \mathrm{dl}$ ). A matched group of normal birth weight (NBW), non-anaemic (NFA) new-borns were enrolled as controls. Morbidity episodes were recorded at 4-weekly intervals and at each extra visit made to a health centre with any illness. Infants in the NBW NFA group experienced an average of 1.15 (95\% C.I. 0.99, 1.31), 1.04 (0.89, 1.19), $0.92(0.73,1.11)$ episodes per year of malaria, respiratory infection and diarrhoea respectively. Corresponding values for the LBW FA group were $0.83(0.5,1.16), 0.82(0.5,1.16)$ and $0.76(0.33,1.19)$. FA was not associated with a higher incidence of morbidity, but was significantly associated with a shorter time to first illness episode $(\mathrm{p}=0.014)$. LBW was not a significant risk factor for higher morbidity incidence. $\mathrm{LBW}$ and FA were not significant risk factors for incidence of illness episodes in infants.

\section{Introduction}

In sub-Saharan Africa, infant morbidity and mortality are excessively high and reductions in mortality rates have not kept pace with expectations, with rates only improving from 114 in 1980, to 108 per 1000 live births in $2000^{1}$. Over the same period, greater reductions have occurred in other regions of the world. In Malawi, infant mortality rates of 157 and 117 per 1,000 live births were reported in 1980 and $2000^{1}$. Most available data indicate that diarrhoea, malaria and acute respiratory infections account for the majority of these infant deaths ${ }^{2}$.

Low birth weight is a risk factor for infant mortality ${ }^{3,4}$. Its association with infant morbidity is less well established and it is unclear whether the increased morbidity risk relates to a higher incidence or to severity of infection. Fetal anaemia is also an important risk factor for anaemia in the first six months of life ${ }^{5}$ and may contribute to mortality risk ${ }^{6}$. The effect of fetal anaemia on infectious infant morbidity has not been studied. As low birth weight and fetal anaemia have been related to malaria in pregnancy ${ }^{7-9}$, it is important to establish their association with infant morbidity in malaria endemic areas. The objective of this study was to investigate the incidence and severity of infectious morbidity in infants in relation to low birth weight and fetal anaemia in an area with high malaria transmission.

\section{Patients and methods}

This study was undertaken between March1993 and September 1995 in a rural area of Southern Malawi, an area of high HIV seropositivity with holo-endemic malaria transmission (10). The estimated population size in 1987 was 316,733 , of whom 68,998 were women of child bearing age. The estimated infant mortality rate in this district was 174 per 1000 live births compared to a national average of 159 deaths per 1000 live births(11). The study was located in Chikwawa District Hospital (CDH) and Montfort Hospital (MH), which are $30 \mathrm{~km}$ apart. All women attending the antenatal facilities of these hospitals between March 1993 and June 1994 were enrolled at their first antenatal visit after informed consent was obtained. At recruitment a questionnaire was completed by a project nurse, which included information on age, literacy and obstetric history. For logistic reasons information on delivery was only collected from women who attended the hospital facilities of $\mathrm{CDH}$ or $\mathrm{MH}$ for delivery. The baby was weighed to the nearest 10 grams on a Salter scale immediately after birth and gestational age was assessed using a modified Ballard method ${ }^{7}$. In previous papers, the study area, methodology and maternal health in relation to malaria, anaemia and HIV are described in more detai ${ }^{15,10}$.

\section{Infant follow-up}

A stratified sample of infants was selected based on low birth weight (LBW) and fetal anaemia (FA). Matched controls were infants born on the same day with normal birth weight (NBW) and no fetal anaemia (NFA). Cases were defined as LBW FA, LBW NFA and NBW FA. Babies who died within 48 hours post-partum, during the hospital observation period, were excluded. To allow for seasonal factors, enrolment occurred over a one-year period. Mothers were asked to return to the hospital with their child at 4-weekly intervals with the first visit occurring at six weeks of age so as to ease integration with the immunisation schedule. An active surveillance system was in place to enhance follow-up for non-attenders. Data was also collected when the child was brought to a health facility with an intercurrent illness at unscheduled visits. If this visit was at a facility outside the study area data was extracted from the child's under-5 health record. At every visit, scheduled or unscheduled, to one of the two study hospitals, research nurses completed a questionnaire which included questions on cough, eye discharge and diarrhoea. In addition, a basic clinical examination was performed including axillary temperature and respiratory rate. Infants presenting with an illness were treated according to Malawi standard management guidelines ${ }^{12}$.

\section{Laboratory investigations}

Maternal blood, collected by venepuncture at recruitment and delivery, and cord blood were assessed for haemoglobin level $(\mathrm{Hb})$ and malaria. Malaria slides were also made from placental blood obtained from deep between the villi. Infant blood for $\mathrm{Hb}$ and malaria assessment was collected by finger prick at scheduled visits at $10,18,26,38$ and 52 weeks and with every 
illness when attending a study hospital. $\mathrm{Hb}$ was measured photometrically after conversion to cyanometheamoglobin using a heamoglobinmeter (Biotron). Malaria slides were stained with Giemsa and read counting asexual Plasmodium falciparum parasites against 200 white blood cells. Maternal HIV status was assessed after obtaining informed consent using two different enzyme-linked immunosorbent assays.

\section{Definitions}

LBW was defined as birth weight less than 2500 grams and FA as cord haemoglobin less than $12.5 \mathrm{~g} / \mathrm{dl}$. This value is two standard deviations below the mean cord $\mathrm{Hb}$ for industrialised countries(8). Morbidity was defined as specific disease episodes reported by the mothers at scheduled visits or unscheduled visits. The clinical diagnoses were established and documented by the Clinical Officers or Medical Assistants attached to the attended health facilities. Fever was defined as axillary temperature greater than $37.5^{\circ} \mathrm{C}$. A respiratory rate greater than 60 per minute was considered a lower respiratory infection for children under two months of age, for older infants, a cut-off value of 50 per minute was used(13). A persistent episode was defined as an illness which lasted between two scheduled visits spanning a period of four weeks.

\section{Sample size}

The sample size calculation was based on detecting a significant difference in prevalence of infant malaria between LBW and NBW infants. To detect a risk ratio of at least 2, with $95 \%$ confidence and $80 \%$ power, with a 6 to 1 ratio of infants in the NBW to LBW group, a sample of $162 \mathrm{NBW}$ and $27 \mathrm{LBW}$ babies was required. This assumed a 30\% infant malaria prevalence among these infants.

\section{Analysis}

Data were analysed using SPSS for windows, version 11.0 (2001) and EPI-Info 2000. Time to first infection was calculated using Kaplan-Meier curves and the Log rank test was used to compare the age at first infection between subgroups. To estimate incidence, episodes of morbidity (including death) at all visits (scheduled and unscheduled) were considered. The follow-up period was the number of days from birth to 1 year of age or last visit, whichever came first. Incidence estimates and their confidence intervals were calculated by dividing the number of new episodes by the duration of follow up and converted to annual rates. Comparative proportions between groups were compared using the chi-square test.

\section{Ethical approval}

The study received ethical approval from the College of Medicine Research and Ethics committee.

\section{Results}

The study recruited 561 infants from 1523 deliveries of whom 43 were twins and $50.3 \%$ were males. Among those recruited, 494 infants attended for at least one scheduled visit (Table 1) and a variable number of unscheduled visits (extra visits). There were 67 babies who did not attend for
Table 1: Number of mothers attending at scheduled visits

\begin{tabular}{|l|l|l|l|}
\hline Weeks after birth & Number & Weeks after birth & Number \\
\hline & & & \\
\hline 6 weeks & 352 & 30 weeks & 302 \\
\hline 10 weeks & 353 & 34 weeks & 306 \\
\hline 12 weeks & 339 & 38 weeks & 302 \\
\hline 18 weeks & 355 & 42 weeks & 306 \\
\hline 22 weeks & 338 & 46 weeks & 290 \\
\hline 26 weeks & 327 & $50-52$ weeks & 324 \\
\hline
\end{tabular}

any visit and they were excluded from any further analysis. There were 112 infants with LBW, 124 with FA, 35 with both $\mathrm{LBW}$ and FA and 199 controls (NBW NFA). Birth weight or cord $\mathrm{Hb}$ status was not known for 24 infants and they were excluded from the analysis (Fig 1). The average number of scheduled visits was 7 per child (range 1-13). There were 1215 extra visits (unscheduled) made by 401 infants, an average of three per child. Thirty-eight infants died during the followup period of whom three in the first months post-partum. There were a total of 4888 months of follow-up for 494 infants, with a median follow-up period of 11 months. Table 2 shows the baseline maternal characteristics of the study groups. Mothers with infants in the LBW FA category had significantly more placental malaria than controls $(p<0.05)$. Mothers of infants in the LBW NFA category were more likely to be anaemic, adolescent, of short stature and more likely to be primiparious than controls (all $\mathrm{p}<0.05$ ).

Table 2: Baseline maternal characteristics (\%) of study group

\begin{tabular}{|l|l|l|l|l|}
\hline $\begin{array}{l}\text { Maternal } \\
\text { Characteristics }\end{array}$ & $\begin{array}{l}\text { LBW FA } \\
(\mathrm{n}=35)\end{array}$ & $\begin{array}{l}\text { LBW NFA } \\
(\mathrm{n}=112)\end{array}$ & $\begin{array}{l}\text { NBW FA } \\
(\mathrm{n}=124)\end{array}$ & $\begin{array}{l}\text { NBW NFA } \\
(\mathrm{n}=199)\end{array}$ \\
\hline Married & 91.4 & 98.2 & $99.2^{* * *}$ & 95.0 \\
\hline Primigravidae & 42.9 & 38.4 & $14.5^{* *}$ & 27.6 \\
\hline Height <150cm & 11.4 & $18.8^{* *}$ & 6.5 & 7.5 \\
\hline $\begin{array}{l}\text { Adolescent } \\
(<20 \text { years) }\end{array}$ & 37.9 & $35.5^{* *}$ & 17.9 & 21.8 \\
\hline Illiterate & 74.3 & 75.9 & 71.8 & 69.8 \\
\hline HIV (+ve) & 31.4 & 26.8 & 25.0 & 25.6 \\
\hline Hb<8g/dl) (delivery) & 17.1 & $21.4^{* *}$ & $15.3^{* * *}$ & 7.0 \\
\hline $\begin{array}{l}\text { Malaria at } \\
\text { recruitment + }\end{array}$ & 31.4 & 19.6 & 16.9 & 23.6 \\
\hline Malaria at delivery + & 28.6 & 29.55 & 29.0 & 24.1 \\
\hline Placental malaria+ & $34.3^{* * *}$ & 19.6 & 21.8 & 16.6 \\
\hline Mean age (yrs. $)$ & 23.6 & 23.4 & 24.6 & 24.7 \\
\hline
\end{tabular}

${ }^{*} p<0.001,{ }^{* *} p<0.01$,

${ }^{* * *} p<0.05$ compared to controls (NBW NFA)

+ parasitaemia

LBW Low birth weight

NBW Normal birth weight

FA Fetal anaemia

NFA No fetal anaemia

\section{Evaluation of clinical diagnosis}

A total of 467 malaria diagnoses were made by Clinical Officers and Medical Assistants of which 338 were made at the study hospitals. Of these, $81.2 \%$ of the infants were febrile and $89.3 \%$ were prescribed treatment with sulfadoxine pyrimethamine. There were $47.3 \%$ with an unknown malaria 
Figure

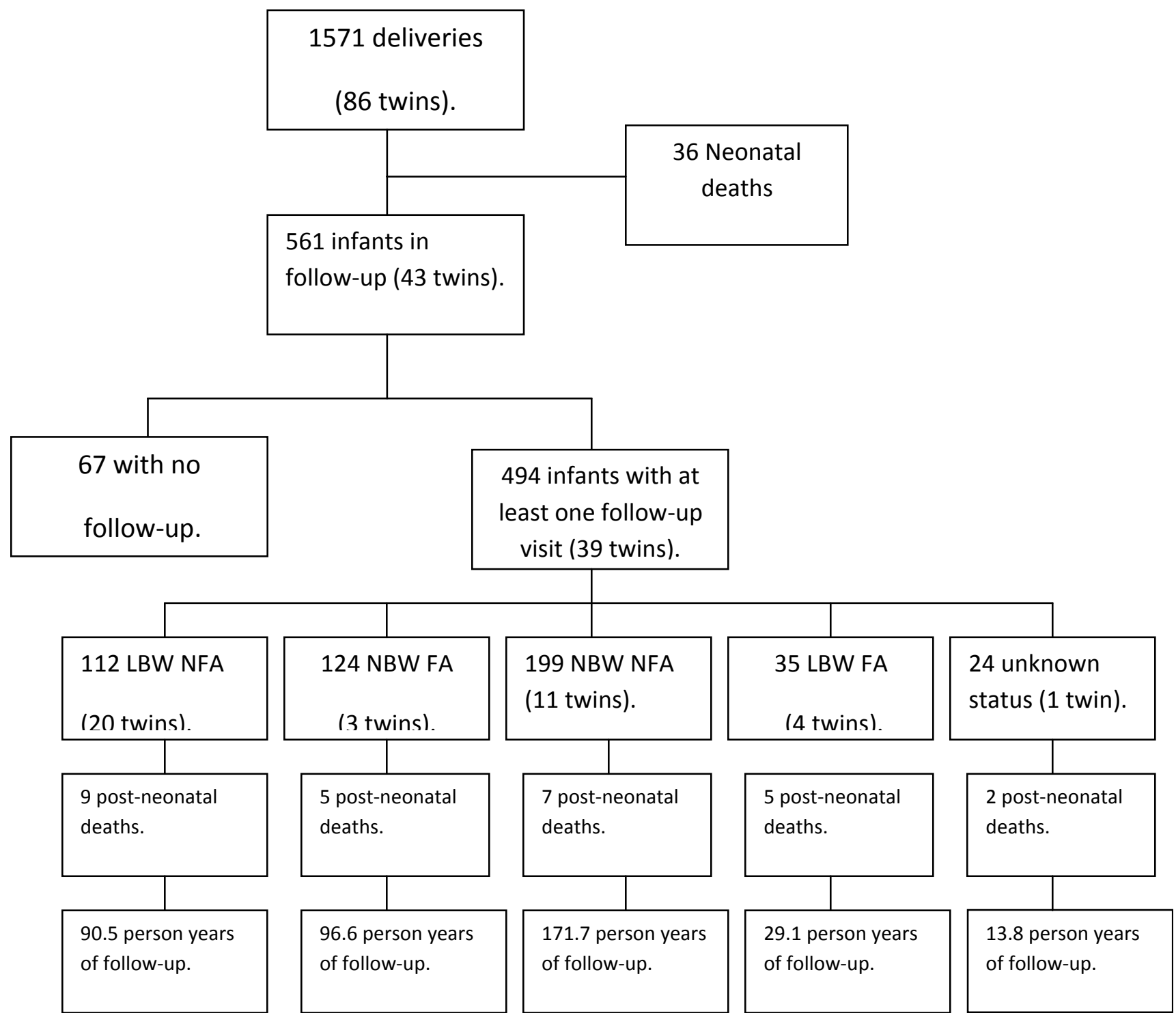

slide result, $21 \%$ with a negative and $31.7 \%$ with a positive malaria slide result. Malaria prevalence at 2 months was $2.3 \%$ and after this had an average of $6.2 \%$ per visit.

There were 440 episodes of respiratory infection recorded of which 245 were made by Clinical Assistants and Medical Officers at study hospitals. Of these, $75.9 \%$ of the infants were febrile and $53.5 \%$ had a malaria slide taken of which $21.4 \%$ were positive. In infants under 2 months, $64.3 \%$ had a lower respiratory tract infection, in infants older than 2 months, $70.2 \%$ had a lower respiratory tract infection. Among those diagnosed with a respiratory infection, $87.2 \%$ of mothers mentioned that their children had a cough and/ or runny nose, $31 \%$ were treated with penicillin and $40.8 \%$ with cotrimoxazole. Of the one hundred infants $(35.5 \%)$ who received SP, 84 also received an antibiotic.

There were a total of 323 diagnoses of diarrhoea of which 241 were made by Clinical Assistants and Medical Officers at study hospitals. $86.6 \%$ of the mothers mentioned to study nurses that their children had watery diarrhoea and $6.9 \%$ bloody diarrhoea. $81.7 \%$ of infants were treated with ORS. Eighty-seven infants had as main diagnosis anaemia. Of these, 78 were at the study hospitals of which $72(92.3 \%)$ had an $\mathrm{Hb}$ of less than $8 \mathrm{~g} / \mathrm{dl}$. Eye infections were the most common diagnosis (494) and 403 were seen at the study hospitals and $90.9 \%$ had eye discharge.

\section{Morbidity incidence}

There were a total of 2221 new episodes of morbidity during the follow-up period. On average an infant experienced 5.45 (95\% C.I. 5.2; 5.68) illness episodes during the first year of life (Table 3). Control group infants experienced 5.34 episodes (95\%C.I. 4.99; 5.69); LBW FA, 4.67 (3.91; 5.68); LBW NFA, 5.81 (5.33; 6.34) and NBW FA, 5.81 (5.34; 6.32) episodes per year. Highest incidence, regardless of study group, occurred for eye infections at $1.23(1.11,1.33)$ episodes per year. This was followed by malaria at $1.14(1.04,1.25)$ episodes per year, respiratory infection $1.08(0.98,1.18)$ and diarrhoea 0.92 $(0.80,1.05)$ episodes per year. Infants in the LBW NFA group had the highest incidence of malaria (1.26) and infants in the LBW FA group the lowest (0.83) although this difference was not significant. The highest incidence of death occurred in infants with LBW and FA. Morbidity incidence for coinfections of malaria, respiratory infection and/or diarrhoea showed that there was no significant difference amongst the four study categories.

\section{Cumulative incidence of first morbidity episodes}

By one month, $10 \%$ and by 12 months $95 \%$ of the infants 
Table 3: Morbidity Incidence per person year by study group

\begin{tabular}{|l|l|l|l|l|}
\hline Infection/llIness & $\begin{array}{l}\text { LBW FA } \\
(n=35)\end{array}$ & $\begin{array}{l}\text { LBW NFA } \\
(n=112)\end{array}$ & $\begin{array}{l}\text { NBW FA } \\
(n=124)\end{array}$ & $\begin{array}{l}\text { NBW NFA } \\
(n=199)\end{array}$ \\
\hline Eye & $1.10(0.72,1.48)$ & $1.22(0.99,1.45)$ & $1.45(1.21,1.69)$ & $1.17(1.01,1.33)$ \\
\hline Malaria & $0.83(0.5,1.16)$ & $1.26(1.03,1.49)$ & $1.13(0.92,1.34)$ & $1.15(0.99,1.31)$ \\
\hline Respiratory & $0.82(0.5,1.16)$ & $1.17(0.95,1.39)$ & $1.20(0.98,1.42)$ & $1.04(0.89,1.19)$ \\
\hline Diarrhoea & $0.76(0.33,1.19)$ & $1.00(0.73,1.27)$ & $0.91(0.66,1.16)$ & $0.92(0.73,1.11)$ \\
\hline Skin & $0.24(0.06,0.42)$ & $0.34(0.22,0.46)$ & $0.31(0.20,0.42)$ & $0.25(0.17,0.33)$ \\
\hline Anaemia & $0.31(0.11,0.51)$ & $0.24(0.14,0.34)$ & $0.18(0.01,0.26)$ & $0.21(0.14,0.28)$ \\
\hline Ear & $0.07(-0.03,0.17)$ & $0.04(0.0,0.08)$ & $0.08(0.02,0.14)$ & $0.10(0.06,0.16)$ \\
\hline Mouth & $0.10(-0.02,0.22)$ & $0.04(0.0,0.08)$ & $0.09(0.03,0.15)$ & $0.05(0.02,0.08)$ \\
\hline Nutritional deficiency & $0.03(-0.04,0.10)$ & $0.02(-0.01,0.05)$ & $0.03(-0.01,0.07)$ & $0.04(0.01,0.07)$ \\
\hline Death & $0.17(0.02,0.32)$ & $0.10(-0.05,0.07)$ & $0.05(0.0,0.10)$ & $0.04(0.01,0.07)$ \\
\hline Other & $0.24(0.06,0.42)$ & $0.33(0.19,0.47)$ & $0.35(0.23,0.47)$ & $0.33(0.23,0.43)$ \\
\hline Total & 4.67 & 5.81 & 5.81 & 5.34 \\
\hline Parenthesis:95\% Confiden
\end{tabular}

parenthesis: $95 \%$ confidence Interval.

had experienced an illness episode (Table 4). Almost $40 \%$ of the infants had had an illness episode by three months with respiratory infections being the most common (17.9\%). First malaria episode occurred in $8.3 \%$ by three months and by six months $34 \%$ had experienced at least one malaria episode. At one year, at least one episode of malaria, respiratory tract infection, diarrhoea and eye infection occurred in over $60 \%$ of the infants. Cumulative incidence in time, stratified by birth weight and fetal anaemia categories, showed no significant difference in incidence between groups.

Table 4 Cumulative incidence of first morbidity episode

\begin{tabular}{|l|l|l|l|l|l|}
\hline Infection/IIness & \multicolumn{5}{|c|}{ Month of follow-up } \\
\hline & 1 & 3 & 6 & 9 & 12 \\
\hline Any (including death) & 0.05 & 0.39 & 0.75 & 0.89 & 0.95 \\
\hline Malaria & 0.01 & 0.08 & 0.34 & 0.55 & 0.68 \\
\hline Respiratory & 0.02 & 0.18 & 0.34 & 0.49 & 0.61 \\
\hline Diarrhoea & 0.01 & 0.08 & 0.29 & 0.50 & 0.65 \\
\hline Anaemia & 0.00 & 0.01 & 0.05 & 0.12 & 0.21 \\
\hline Eye & 0.01 & 0.12 & 0.37 & 0.53 & 0.66 \\
\hline Skin & 0.01 & 0.04 & 0.11 & 0.16 & 0.26 \\
\hline Mouth & 0.01 & 0.01 & 0.02 & 0.04 & 0.06 \\
\hline Ear & 0.01 & 0.01 & 0.03 & 0.05 & 0.07 \\
\hline Other & 0.02 & 0.05 & 0.11 & 0.18 & 0.28 \\
\hline
\end{tabular}

\section{Time to first illness episode}

Figure 2 shows the survival curves for time to first illness episodes for the four study groups. There was a significantly shorter time to first illness episode for infants with NBW FA than for those with LBW NFA $(p=0.002)$, LBW FA $(\mathrm{P}=0.004)$, or controls $(\mathrm{p}=0.014)$. Infants with LBW FA had a longer period to first malaria episode but this difference was not significantly different from the other groups.

\section{Persistent episodes}

There were 66 infants who experienced persistent episodes covering two scheduled visits. These episodes comprised $44.6 \%$ with respiratory infection, $16.2 \%$ with malaria and
8.1\% with diarrhoea. In the LBW FA group, 17.1\% of infants experienced persistent episodes compared to $17.0 \%$ in the NBW FA, $13.1 \%$ in the NBW NFA and $9.0 \%$ in the LBW NFA group. Prevalence of persistent episodes did not significantly differ between groups.

\section{Hospital Admissions}

Altogether, 71 infants (14.4\%) were admitted in hospital and 7 were admitted twice. Among infants admitted, ${ }^{13}(16.7 \%)$ had gastro-enteritis, ${ }^{22}(28.2 \%)$ pneumonia, $20(25.6 \%)$ malaria, ${ }^{2}(2.6 \%)$ nutritional deficiency, $2(2.6 \%)$ meningitis, $1(1.3 \%)$ tuberculosis, and $18(23.8 \%)$ other illnesses. Nine (12.7\%) of the admitted infants died. The primary diagnoses in the children who died were gastro-enteritis (2), pneumonia (1), meningitis (2), nutritional deficiency (1) or other illnesses (3). In the LBW FA group, $14.3 \%$ of the infants were admitted at least once. The corresponding figures were $16.1 \%$ in both the NBW FA and LBW NFA groups and $11.6 \%$ in the control group. The prevalence of admissions in the different groups was not significantly different.

\section{Discussion}

This study achieved good follow-up with reporting rates at scheduled visits of over $60 \%$. Only 67 infants did not attend once for follow-up mainly due to long residential distance from the hospital. Infants experienced on average 5.45 illness episodes per year. This is within the range of 2.3 to 6.5 illness episodes per year reported ${ }^{14}$ for children in Kwara State Nigeria, rural Ethiopia and Burkina-Faso. Follow-up times ranged from 171.7 person years in controls to 29.1 person years in the LBW FA group which was due to the small study number $(n=35)$ in that group. This difference could be a source of confounding as shorter periods may incur fewer new episodes. A difference between our study and that of Vaahtera ${ }^{14}$ was that we considered morbidity as episodes reported by the mother and assessed by Clinical Officers and Medical Assistants while they reported morbidity as episodes recalled (fortnightly) by the mother in the community. The present study therefore reports mostly on more severe forms of morbidity. 


\section{Malaria diagnosis}

Almost two thirds of infants with a diagnosis of malaria had a positive slide result. Sulphadoxine-pyrimethamine (SP) was prescribed to the majority of malaria cases $(89.3 \%)$ indicating good adherence to the Malawi National Malaria Control Programme guidelines for recommended treatment of febrile children ${ }^{15}$ as well as the current Integrated Management of Childhood Illnesses (IMCI) guidelines ${ }^{13}$. The usefulness of diagnostic algorithms for managing malaria in children on the basis of such guidelines has been reviewed and they show good sensitivity and specificity, although most studies have only included children older than one year ${ }^{16}$. As $39.9 \%$ of infants with a malaria diagnosis were slide negative, then to considerable over-use of SP would occur. In addition, at least $10 \%$ of malaria cases would be missed if diagnosis on the basis of fever alone was used. Afolabi et. al reported that the sensitivity of malaria clinical diagnosis in Nigerian infants below 6 months was $32.7 \%$ (compared to microscopy) and specificity was $74.6 \%{ }^{17}$. The IMCI guidelines for treating all febrile illnesses as malaria offer a reasonable strategy for diagnosing infant malaria in febrile children. However, of the 245 respiratory infection episodes, $75.9 \%$ were febrile, of whom only $21.2 \%$ had a positive malaria slide, and all of these would have received anti-malarial treatment unnecessarily if IMCI guidelines were followed.

\section{Morbidity incidence}

Respiratory infection, malaria and diarrhoea are the commonest form of morbidity in infants in Africa and elsewhere in the developing world ${ }^{18-20,2}$. The incidence of respiratory infections in infants in Michigan (USA) and Costa Rica were higher than in the present study with annual rates of 6.1 and $3.3^{21,22}$. Surveillance in these studies was weekly or fortnightly compared to 4-weekly in the present study. In a 10 week interval surveillance in Nigeria, an annual incidence of 4.9 per year of malaria episodes in infants estimated using a Markov chain model was reported ${ }^{23}$. Differences in levels of malaria endemicity limit comparisons between these studies. A further Malawi study which used monthly surveillance reported comparable incident rates to those observed in the present cohort $^{13}$.

Diarrhoea was uncommon in the first three months of life, with a 3 -fold increase by 6 months. This may partly relate to the loss of passively acquired maternal immunity, but increasing incidence of diarrhoea in infancy also relates to the introduction of water and weaning foods prepared using unhygienic practices ${ }^{24}$. Persistent episodes are associated with early weaning ${ }^{25}$ and in this population $17 \%$ of the infants were already given a local infant food (phala) by 6 weeks of age. This figure increased to $42 \%$ by 12 weeks of age.

Eye infections were the most common form of morbidity Prevalence of active trachoma in children in the lower Shire valley was 36.7\% in 1983 and 14\% in 1999 (Courtright, 1999 , unpublished results). The present findings indicate that eye infections are a significant problem from early in infancy and appropriate recognition of their significance will be important in order to establish suitable awareness in the community and in order to improve prevention strategies.

\section{Fetal anaemia and morbidity incidence}

The infants with NBW and FA had a significantly higher cumulative incidence of morbidity and a shorter time to first illness episode compared to the other study groups. Infants with FA also had more persistent episodes although this was not statistically significant. Fetal anaemia has previously been reported to be associated with infant anaemia in the first six months of life and with increased mortality in this cohort ${ }^{5,6}$. The causes of FA have not been determined but may result from maternal iron deficiency anaemia and dense placental malaria infections, both of which commonly occur in these mothers. The evidence that severe childhood anaemia is an important risk factor for child mortality has recently been reviewed ${ }^{26}$.

\section{Birth weight and morbidity incidence}

In this study, LBW was not identified as a significant risk factor for disease incidence. A number of further studies have also reported that LBW was not risk factor for infant morbidity. In the USA, no differences in frequency of diarrhoea episodes between LBW and NBW infants was found ${ }^{27}$. In the same study, gastrointestinal diseases were less frequent among LBW children (25.9 episodes versus 30.1 episodes per 100 children). In a cohort of children in Guatemala, it was also reported that reduced birth weight showed no consistent relation to infection, or to cumulative clinical manifestations ${ }^{25}$. In a study of malaria infection in early infancy in Malawi, birth weight was not associated with a positive blood slide in the first three months of life ${ }^{28}$.

However, other studies do report an association of LBW with infant morbidity. In the, United Kingdom, Acheson ${ }^{29}$ reported that babies weighing less than 2,490 grams experienced significantly higher morbidity than NBW babies. $\mathrm{Knobloch}^{30}$ found that LBW infants in Maryland, USA had $50 \%$ more illness episodes than NBW infants. Ashworth ${ }^{31}$ also reported an association of LBW with pneumonia risk in a review of studies from different countries and showed there was an increased risk of diarrhoea in LBW infants. In a small study of paediatric diarrhoea in Sri Lanka, good evidence for an association between LBW and increased risk of diarrhoea was found ${ }^{32}$. In a study from north east Brazil, LBW infants had increased risk of diarrhoea but not respiratory infection ${ }^{33}$. These different results between studies may partly relate to methodological issues ${ }^{27}$. In general, there is insufficient data on the relative risk of morbidity by birth weight categories to allow comparative computations for morbidity associations to be made ${ }^{34}$. There are also very few studies on this from Africa, especially from malaria and HIV endemic areas.

\section{Severity of illness/persistent episodes}

A number of factors may confound incidence estimates including disease severity. More severe infections may occur in LBW babies because of the association of LBW with impaired immune function ${ }^{35-37}$. In the present study, the highest prevalence of persistent episodes occurred in the LBW FA group although this was not statistically significant. The occurrence of pre-term or growth retarded new-borns may have independent effects on morbidity risk. In this cohort, $32.6 \%$ of babies had a LBW of whom $45.6 \%$ were premature and $54.4 \%$ were intra-uterine growth retarded. A further confounding factor is maternal anaemia. Mothers of infants in the study groups were significantly more anaemic than controls. HIV prevalence among pregnant mothers was $25.6 \%(10)$, although this did not vary significantly between 
the four groups. Paediatric HIV/AIDS would confound the effect of birth weight and fetal anaemia on infant morbidity as between $20 \%$ to $43 \%$ of infants may have become infected through mother to child transmission ${ }^{38-40}$.

In conclusion, this study has shown that LBW and FA were not significant risk factors for morbidity incidence in infants, although babies with FA experienced their first infection at an earlier age and were more likely to have a persistent morbidity episode. Interventions to reduce infant morbidity need to address risk factors for severity of infectious episodes in order to optimise care for those babies.

\section{Acknowledgements}

We are grateful for financial support provided by the Gates Malaria Partnership (GMP) and the European Commission Programme for Life Sciences and Technologies for Developing Countries to collect the and analyse the data.

\section{References}

1. United Nations Children's Fund, Unicef Statistics, Child Mortality 2002.

2. http://www.childinfo.org/cmr2.html (accessed 28 June 2003).

3. World Health Organisation, (1998). The World Health Report 1998. Life in the 21st Century, a Vision for All. Geneva, WHO.

4. Wilcox, J.A. (2001). On the importance - and the unimportance - of birth weight. International Journal of Epidemiology; 30:1233-1241.

5. McCormick M.C. (1985). The contribution of low birth weight to infant mortality and childhood morbidity. N. Engl. J Med. , 312:8290 .

6. le Cessie S., Verhoeff F.H., Mengistie G., Kazembe P., Broadhead R., Brabin B.J. (2002). Changes in haemoglobin values among infants in Malawi: the effect of low birth weight and fetal anaemia. Arch Dis Child Fetal Neonatal Ed . 86(3):182-7.

7. Verhoeff, F.H (2000). Malaria in pregnancy and its consequences for the infant in rural Malawi. Ph.D. Thesis, University of Leiden.

8. Verhoeff, F.H., Brabin, B.J., Chimsuku, L., Kazembe, P., Broadhead, R.L. (1999). Malaria in pregnancy and its consequences for the infant in rural Malawi. Ann Trop Med Parasitol;93:S25-S33.

9. Brabin, B.J.(1992) Fetal anaemia in malarious areas: its causes and significance. Ann. Trop Paed, 12, 303:-310.

10. Greenwood, A. M., Armstrong, J. R., Byass, P., Snow R., Greenwood B. M.(1992). Malaria chemoprophylaxis, birthweight and child survival. Transactions of the Royal Society of Tropical Medicine and Hygiene, 86, 483-485.

11. Verhoeff, F.H., Brabin B.J., Hart C.A., Chimsuku, L., Kazembe, P., Broadhead, R. (1999). Increased prevalence of malaria in HIV infected pregnant women and its implications for malaria control. Tropical Medicine and International Health. 4: 5-12.

12. Malawi Population and Housing Census (1987). National Statistical Office (NSO). Summary of Final Results, volume1, p53. Zomba, Malawi.

13. Phillips, J.A., Kazembe, P.N., Nelson, E.A.S, Fisher, J.A.F., Grabosch, E. (1998). A Paediatric Handbook for Malawi. Montfort Press, Limbe, Malawi.

14. World Health Organisation, (2000). Handbook. IMCI. Integrated Management of Childhood Illness.

15. Vaahtera, M., Kulmala, T., Maleta, K., Cullinan, M., Salin, L., \& Ashorn, P.(2000) Epidemiology and predictors of infant morbidity in rural Malawi. Paediatric \& Perinatal Epidemiology, 14, 4,363-371.

16. Ministry of Health, Malawi (1992). Malawi Guidelines for the Management of Malaria. Malaria Control Programme, Community Health Sciences Unit, Lilongwe, Malawi.

17. Chandramohan, D., Jaffar, S., Greenwood, B. (2002). Use of clinical algorithms for diagnosing malaria. Tropical Medicine and International Health, 7,1: 45-52.

18. Afolabi, B.M., Salako, L.A., Mafe, G., Ovwigho, U.B., Rabiu, K.A., Sanyaolu, N.O., Ibrahim, M.M. (2001). Malaria in the first 6 months of life in urban African infants with anaemia. Am. J. Trop. Med. Hyg. 65(6): 822-827.
19. Lindskog, U., Bjorksten, B., Gebre-Medhin, M.(1994). Infant care in rural Malawi. A prospective study of morbidity and growth in relation to environmental factors. Ann Trop Paediatr. 14(1):37-45.

20. Jaffar, S., Leach, A., Greenwood, A.M., Jepson, A., Muller, O., Ota, M., Bojang, K., Obaro, S., Greenwood, B.M.(1997). Changes in the pattern of infant and childhood mortality in upper river division, The Gambia, from 1989-1993. Trop Med Int Health, 2(1):28-37.

21. Kolsteren, P.W, Kusin, J.A., Kardjati, S. (1997). Morbidity and growth performance of infants in Madura, Indonesia. Ann. Trop. Paediatr, 17(3):201-8

22. Monto, A.S., Ullman, B.M. (1974). Acute respiratory illness in an American Community. JAMA; 227(2):164-69.

23. Smith, T.A., Lehmann, D., Coakley, C., Spooner, V., Alpers, M.P. (1991). Relationships between growth and acute lower-respiratory infections in children aged $<5 \mathrm{y}$ in a highland population of Papua New Guinea. Am. J. Clin. Nutr; 53:963-70.

24. Bekessy, A., Molineaux, L., Storey, J. (1976). Estimation of incidence and recovery rates of plasmodium falciparum parasitaemia from longitudinal data. Bull. Wld. Hlth. Org. 54:685-693.

25. Gordon, J.E., Chitkara, I.D., Wyon, J.B. (1963). Weanling Diarrhoea. Am. J.Med. Sci.245: 345-377.

26. Mata, L.J., Urrutia, J.J., Gordon, J.E. (1967). Diarrhoeal disease in a cohort of Guatemalan village children observed from birth to age tow years. Trop. Geogr. Med., 19:247-257.

27. Brabin, B.J., Premji, Z., Verhoeff, F. (2001). An analysis of anaemia and child mortality. The Journal of Nutrition. 131, 2S-II; 636S-648S.

28. Van de Berg, B.J. (1968). Morbidity of low birth weight and/or preterm children compared to that of the "mature". 1. Methodological considerations and findings for the first 2 years of life. Pediatrics, 42(2):591-597.

29. Slutsker, L., Khoromana, C.O., Hightower, A.W., Macheso, A., Wirima, J.J., Breman, J.G., Heymann, D.L., Steketee, R.W. (1996). Malaria infection in infancy in rural Malawi. Am. J. Trop. Med. Hyg., 55(1):71-76.

30. Acheson, E.D.(1965). Hospital morbidity in early life in relation to certain maternal and fetal characteristics and events at delivery. British Journal of Preventive and Social Medicine, 19:164-173.

31. Knobloch, H., Pasamanick, B., Harper, P.A., Rider, R.V. (1959). The effect of prematurity on health and growth. American Journal of Public Health. 49:1164-1959.

32. Ashworth, A. (1998). Effects of intrauterine growth retardation on mortality and morbidity in infants and young children. European Journal of Clinical Nutrition. Suppl 1:S34-41.

33. Mertens, T.E., Cousens, S.N., Feachem, R.G. (1986). Evidence of a prolonged association between low birth weight and paediatric diarrhoea in Sri Lanka. Transactions of the Royal Society of Tropical Medicine and Hygiene. 81:196.

34. Lira, P.I., Ashworth, A., Morris, S.S. (1996). Low birth weight and morbidity from diarrhoea and respiratory infection in northeast Brazil. Journal of Pediatrics, 128(4):497-504.

35. Ashworth, A., Feachem R.G. (1985). Interventions for the control of diarrhoeal diseases among young children: prevention of low birth weight. Bull. Wld. Hlth. Org. 63(1):165-184.

36. Chatrath, R., Saili, A., Jain, M., Dutta, A.K. (1997). Immune status of full-term small-for-gestational age neonates in India. J. Trop Pediatr., 43(6):345-348.

37. Chandra, R.K. (1997). Nutrition and the immune system: an introduction. Am. J. Clin. Nutr. 66(2): 460S-463S.

38. Waterlow J.C. (1993). Protein Energy Malnutrition. Edward Arnold. London, UK.

39. Taha, T.E., Justesen, A., Peterson, K., Mtimanalye, L.A., Munthali, P., Canner, J.K., Broadhead, R.L., Chiphangwi, J.D., Miotti, P.G., Bigger, R.J. (1994). An intervention to reduce the risk of mother-to-infant transmission of HIV: results of a pilot toxicity study. East African Medical Journal. 71(11): 712-715.

40. Hira, S.K., Kamanga, J., Bhat, G.J., Tembo, G., Luo, N., Perine, P.L. (1989). Perinatal transmission of HIV-1 in Zambia. British Medical Journal. 299:1250-1252.

41. Bobat, R.(1999). Mortality in a cohort of children born to HIV-1 infected women in Durban, South Africa. South African Medical Journal. 89(6):646-648 